\title{
Pulmonary and diaphragmatic pathology in collagen type I a1 mutant mice with osteogenesis imperfecta
}

\author{
Carolyn J Baglole ${ }^{1}$, Feng Liang ${ }^{1}$, Hussein Traboulsi ${ }^{1}$, Angela Rico de Souza ${ }^{1}$, Christian Giordano ${ }^{1}$, Josephine T Tauer ${ }^{2}$, \\ Frank Rauch ${ }^{2}$ and Basil J Petrof ${ }^{1}$
}

BACKGROUND: Osteogenesis imperfecta (Ol) is most often caused by mutations in type I collagen genes. Respiratory complications have been largely attributed to spine and ribcage deformities. We hypothesized that direct involvement of the pulmonary parenchyma and/or diaphragm by the disease may occur.

METHODS: In Colla1 ${ }^{\mathrm{Jrt} /+}$ mice, a model of severe dominant $\mathrm{Ol}$, mean linear intercept length $(\mathrm{Lm})$ was used to assess the distal airspace size. Cross-sectional area (CSA) and myosin heavy chain $(\mathrm{MyHC})$ phenotype of the diaphragm muscle fibers, as well as contractile properties, were determined. OI mice were also treated with neutralizing antibodies against transforming growth factor- $\beta$ (TGF- $\beta$ ).

RESULTS: Distal airspace enlargement occurred in $\mathrm{Ol}$ mice ( $\mathrm{Lm}+27 \%)$. Diaphragmatic thickness and fiber number were reduced, with increases in fast-twitch type $\|x /\| b$ MyHC fibers. Ex vivo force generation (normalized for CSA) of the diaphragm was also significantly reduced. The increased $L m$ values found in OI mice were not prevented by anti-TGF- $\beta$ antibody treatment.

CONCLUSIONS: The Colla1 $1^{\mathrm{Jt} / \mathrm{t}}$ mouse model of Ol demonstrates: (1) pulmonary airspace enlargement not driven by TGF- $\beta$; and (2) reduced muscle mass and intrinsic contractile weakness of the diaphragm. These results suggest a complex and multifaceted basis for respiratory complications in $\mathrm{Ol}$ that cannot be solely attributed to bone manifestations.

O steogenesis imperfecta (OI) is the most common heritable bone fragility disorder, impacting approximately 1 in 20,000 births and leading to frequent fractures as well as bone deformities (1). Both dominant and recessive forms of the disease exist, which are caused by mutations in a number of genes that are critically involved in collagen synthesis and metabolism $(1,2)$. The most frequent genetic defects are the dominant mutations in COL1A1 or COL1A2 genes coding for collagen type I $\alpha$-chains, which are associated with several classes of disease ranging from mild to severe in their bone manifestations (2).
Respiratory insufficiency has been identified as a leading cause of death in OI patients (3-5). The respiratory complications associated with OI are widely assumed to be due to pulmonary restriction resulting from major spinal deformities. However, there are few lung function data available for OI patients, and their accurate interpretation has been confounded by technical measurement difficulties and uncertainty regarding the most appropriate predicted values to be used in this setting (4). Furthermore, an abnormal decline in pulmonary function has been described in OI children even in the absence of substantial scoliosis (6), suggesting that other mechanisms are involved.

Intriguingly, cartilage-associated protein (CRTAP) geneknockout mice (modeling a rare recessive form of OI) show an evidence of abnormal distal airspace enlargement on necropsy $(7,8)$. In this model, abnormal activation of TGF- $\beta$ signaling has been identified as an important pathogenic mechanism, as indicated by the fact that treatment with antiTGF- $\beta$-neutralizing antibody mitigated both the bone disease and the pathological changes found in the lungs (8). In addition, it has been reported that patients with the most common form of OI (type I) caused by mutations in COL1A1 or COL1A2 show an evidence of impaired skeletal muscle function, consisting of reductions in both the muscle mass and the force generated per unit of muscle cross-sectional area (CSA) (9). These latter findings raise the possibility that OI may also adversely affect the function of the respiratory muscles such as the diaphragm, thus entailing an additional risk for the development of respiratory insufficiency.

Accordingly, the primary objective of the current study was to determine whether pulmonary parenchymal disease or diaphragm muscle pathology are present in Colla $1^{\mathrm{Jt} / \mathrm{t}}$ mice, a mouse model of severe dominant OI that was generated through a mutagenesis screen and harbors a splice site mutation in exon 9 of the Colla1 gene (10). COL1A1 is the most frequently implicated gene in humans with OI (2). In addition, a secondary objective of our investigation was to determine whether anti-TGF- $\beta$ treatment alters the pulmonary phenotype in Colla1 ${ }^{\mathrm{Jt} /+}$ mice.

${ }^{1}$ Meakins-Christie Laboratories and Respiratory Division, McGill University, Montreal, Quebec, Canada; ${ }^{2}$ Shriners Hospital for Children and McGill University, Montreal, Quebec, Canada. Correspondence: Basil J. Petrof (basil.petrof@mcgill.ca)

Received 23 October 2017; accepted 14 February 2018; advance online publication 9 May 2018. doi:10.1038/pr.2018.36 


\section{METHODS}

\section{Animals}

The OI mice (Colla1 $1^{\text {Jrt/+ }}$, gift from Dr J Aubin) and their wild-type (WT) littermates were identified from tail-extracted genomic DNA, which was subjected to PCR as per standard procedures. This dominant mutation results in the skipping of exon 9 with a predicted 18 -amino-acid deletion within the $\mathrm{N}$ terminus of the triple helical domain of the collagen type I $\alpha 1$-chain (10). Male WT and OI mice were initially characterized at 14 weeks of age ( $n=6$ per group). A separate group of female WT $(n=9)$ and OI $(n=7)$ mice were treated with either anti-TGF- $\beta$-neutralizing antibody 1D11 (a generous gift from Genzyme, Cambridge, MA, USA) or a control antibody of the same isotype (13C4) for a total of 4 weeks, starting at 4 weeks old. The antibodies (dose: $10 \mathrm{mg} / \mathrm{kg}$ body mass) were administered three times per week by intraperitoneal injection. After treatment, the mice were killed and the lungs were processed for histological analysis as outlined below. All experimental procedures received approval from the local institutional animal care committees and were conducted in accordance with guidelines of the Canadian Council on Animal Care.

\section{Lung Histology}

Mice were anesthetized with isoflurane and killed by exsanguination. A cannula was inserted into the trachea and tied with a ligature. Lungs were then inflated by the infusion of fixative $10 \%$ formalin, at a constant fluid pressure of $25 \mathrm{~cm} \mathrm{H}_{2} \mathrm{O}$ for $5 \mathrm{~min}$. Lungs and heart were removed en bloc and placed in $50 \mathrm{ml}$ tubes containing $10 \%$ formalin for at least $24 \mathrm{~h}$. The lungs were then dehydrated in $70 \%$ ethanol and placed in paraffin. Fixed lung tissues were oriented with the ventral surface facing down to ensure consistent and uniform inclusion of all lung lobes. Sections of $5 \mu \mathrm{m}$ ( 2 per mouse) were cut and placed on glass slides for staining with hematoxylin and eosin $(\mathrm{H} \& \mathrm{E})$ or, in some cases, for collagen staining by Sirius red. Using light microscopy, 20 histological fields per lung were obtained at a total magnification of $\times 20$. These images were captured to the computer and projected onto a standardized reference grid of horizontal and vertical lines. For each line of the grid projected upon the lung section image, the number of intercepts of the line with alveolar walls was counted. The average alveolar airspace size (represented by the mean linear intercept length or $\mathrm{Lm}$, in $\mu \mathrm{m}$ ) was then calculated by dividing the total grid length by the number of alveolar wall-grid line intersections as described previously $(11,12)$.

\section{Diaphragm Fiber Typology and Size Determination}

Excised diaphragmatic bundles obtained from the mid-costal portion of the muscle were quickly frozen in liquid nitrogen-cooled 2methylbutane and stored at $-80^{\circ} \mathrm{C}$. Cryostat sections $(8 \mu \mathrm{m}$ thin) were then fixed in acetone at $-20^{\circ} \mathrm{C}$, air dried, and blocked at room temperature with a Dako protein block (Dako, X0909, Santa Clara, CA, USA) for $60 \mathrm{~min}$. Subsequently, sections were reacted with primary antibodies (all from Developmental Hybridoma Bank), which were raised against the slow-twitch type I (BA-D5, 1:25), as well as the fast-twitch type IIa (SC-71, 1:300) and type IIx (6H1, 1:25) isoforms of myosin heavy chain (MyHC). By exclusion, diaphragm myofibers that did not exhibit staining for any of the above MyHC isoforms were considered as being type IIb fibers (13). The primary antibodies were mixed in the same antibody diluent (Dako, S3022) and incubated with the muscle sections for $120 \mathrm{~min}$ at room temperature. After three washes in phosphate-buffered saline, the sections were incubated with a secondary antibody (all from Invitrogen, Carlsbad, CA, USA) cocktail containing anti-IgG2b 350 (A21140, 1:500), anti-IgG1 488 (A21121, 1:500), and anti-IgM 555 (A21426, 1:500) for $60 \mathrm{~min}$ at room temperature. After another three washes in phosphate-buffered saline, the sections were mounted using ImmuMount (Thermo, Waltham, MA, USA), and visualized under a Zeiss Axio Imager.M2 fluorescence microscope (Zeiss, Oberkochen, Germany). Images of the entirety of a single mid-costal diaphragm section were acquired with an AxioCam MRm camera and stitched together using Zen Blue Software (Zeiss, Oberkochen, Germany).
The quantitative analysis of $\mathrm{MyHC}$ fiber type proportions as well as the CSA of the individual fibers were determined on at least 200 fibers per sample using ImageJ (National Institutes of Health, Bethesda, MD, USA) (14). A subset of diaphragms was stained with H\&E or Sirius red. Diaphragm thickness was assessed by averaging the perpendicular distance between the thoracic and the abdominal surfaces of the diaphragm.

\section{Evaluation of Diaphragm Contractility}

Diaphragm strips were dissected from the mid-costal portion of the muscle and placed into equilibrated $\left(95 \% \mathrm{O}_{2}-5 \% \mathrm{CO}_{2} ; \mathrm{pH} 7.38\right)$ Krebs solution, as described previously (15). After attaching the muscle to a force transducer/length servomotor system (model 300B; dual mode; Cambridge Technology, Watertown, MA), the optimal length $\left(L_{\mathrm{o}}\right)$ was determined. The force-frequency relationship was measured by sequential supramaximal stimulation for $1 \mathrm{~s}$ at 10,30 , 50,100 , and $150 \mathrm{~Hz}$, with 2 min difference between each stimulation train. To assess fatigue resistance, muscles were intermittently stimulated (330 ms trains at $30 \mathrm{~Hz}$ each second) and the decrement in force production was continuously recorded over a period of $160 \mathrm{~s}$. Muscle force was normalized to total diaphragm muscle strip CSA to derive specific force, which is expressed as $\mathrm{N} / \mathrm{cm}^{2}$.

\section{Statistical Analysis}

Data are expressed as mean values $\pm \mathrm{SD}$. The significance of the differences between the WT and OI groups was analyzed with Student's unpaired $t$ test or two-way ANOVA with a post hoc application of the Tukey's test to adjust for multiple comparisons when appropriate (GraphPad Prism, Software, La Jolla, CA, USA). The Fisher's exact test was used to determine significant differences in proportions. Statistical significance was set at $P<0.05$ for all tests.

\section{RESULTS}

\section{OI Mice Exhibit Alveolar Airspace Enlargement}

At 14 weeks of age, the mean body weight of the OI mice was significantly reduced compared with their WT littermates $(22.45+0.66 \mathrm{~g}$ vs. $29.65+0.82 \mathrm{~g}, \quad P<0.001)$, as described previously (10). As shown by the H\&E-stained lung sections in Figure 1a-d, there were areas of obvious alveolar airspace enlargement in the OI mice, whereas collagen staining by Sirius red (Figure 1e,f) appeared similar between the two groups. To determine the average size of distal airspaces in OI and WT mice, Lm was quantified. As indicated in Figure 1g, there was a significant increase $(+27 \%)$ in the $\mathrm{Lm}$ value in lungs of OI mice compared with their WT littermate controls.

\section{OI Mice Show Alterations in Diaphragm Structure}

Representative diaphragm sections stained with H\&E (Figure 2a,b), Sirius red (Figure 2c,d), and MyHC (Figure 2e,f) are shown. As quantified in Figure $2 \mathrm{~g}$, there was a substantial decrease in the diaphragmatic thickness in the OI group, which amounted to a mean reduction of $\sim 28 \%$. This reduced diaphragmatic thickness was associated with a significant decrease in the average number of muscle fibers spanning the thoracic to abdominal surfaces of the muscle (Figure $2 \mathbf{h}$ ).

For all diaphragm fibers taken together without regard for MyHC phenotype, the mean myofiber size (represented by CSA) demonstrated a trend toward smaller fibers in OI mice (by $\sim 16 \%$ ), but this did not achieve statistical significance (Figure 3a,b). Similarly, analyses of the myofiber CSA data as a function of the different MyHC phenotypes did not reveal 

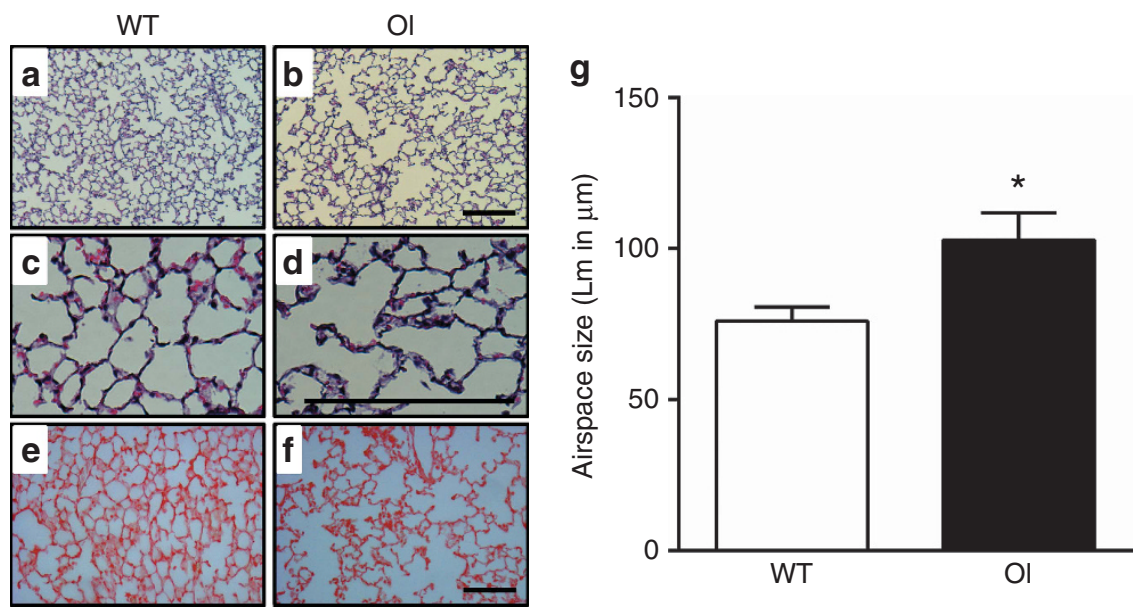

Figure 1. Pulmonary airspace enlargement in osteogenesis imperfecta (OI) mice. Representative lung section images stained with hematoxylin and eosin (H\&E) at different magnifications (a-d) and Sirius red $(\mathbf{e}, \mathbf{f})$; scale bars $=100 \mu \mathrm{m}$. Airspace size quantified by measuring $\mathrm{Lm}(\mathbf{g})$ in the wild-type (WT) and Ol mice ( $n=6$ per group at 14 weeks of age). Values are mean+SD. ${ }^{*} P<0.05$ for WT vs. OI.
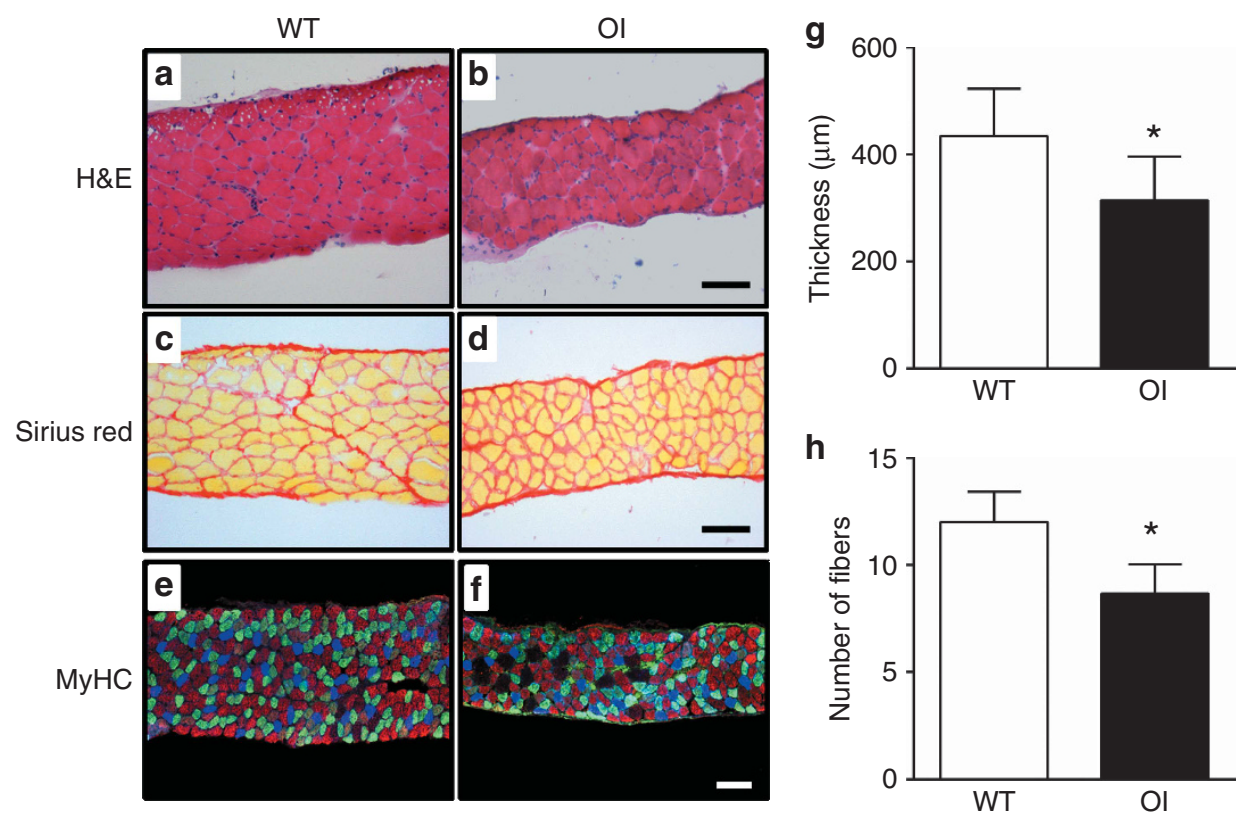

Figure 2. Reduced diaphragm mass in osteogenesis imperfecta (OI) mice. Representative diaphragm section images stained with hematoxylin and eosin (H\&E) (a and b), Sirius red (c and d), and MyHC (e and f); scale bars $=100 \mu \mathrm{m}$. For MyHC staining, antibodies were directed against type I (blue), type lla (green), and type Ilx (red) isoforms. Diaphragm muscle thickness (g) and the number of myofibers spanning the thoracoabdominal surfaces of the muscle $(\mathbf{h})$ were measured $(n=6$ per group at 14 weeks of age). Values are mean+SD. $* P<0.05$ for wild-type (WT) vs. OI.

significant differences between the OI and WT groups (Figure 3c). However, the relative proportions of these different fiber types were mildly altered in the diaphragms of OI mice, consisting of small increases in the percentages of type IIx and IIb fibers, which occurred at the expense of the type IIa population (Figure 3d).

\section{OI Mice Demonstrate Impaired Diaphragm Contractility}

In addition to reduced muscle mass, muscle weakness can be caused by a decrease in the force generation per unit CSA (known as specific force) or an increased susceptibility to muscle fatigue. Therefore, we assessed the specific isometric force produced by isolated diaphragmatic bundles from OI and WT mice in vitro. In OI group diaphragms, there was a modest but statistically significant reduction in specific force production relative to the WT group (Figure 4a). The percent decrement in force production with repetitive stimulation (relative to baseline) did not differ between the OI and WT diaphragms, thus suggesting a similar level of intrinsic resistance to fatigue (Figure $\mathbf{4 b}$ ). 


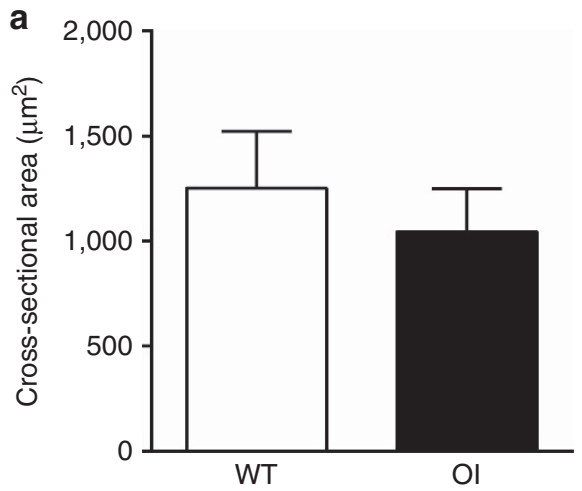

b

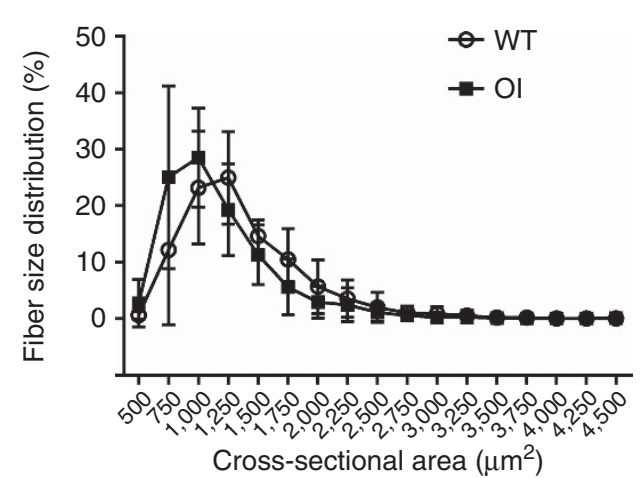

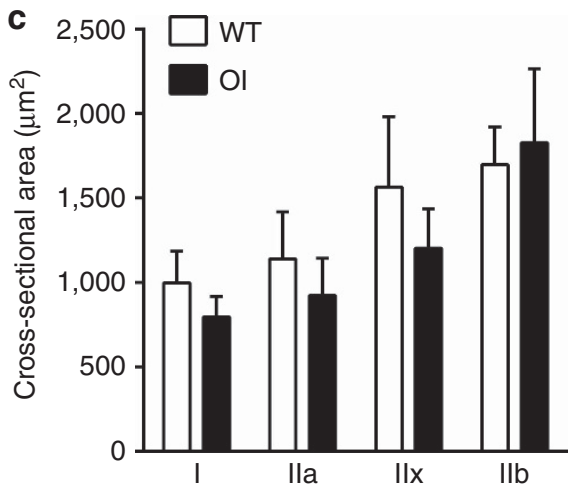

d

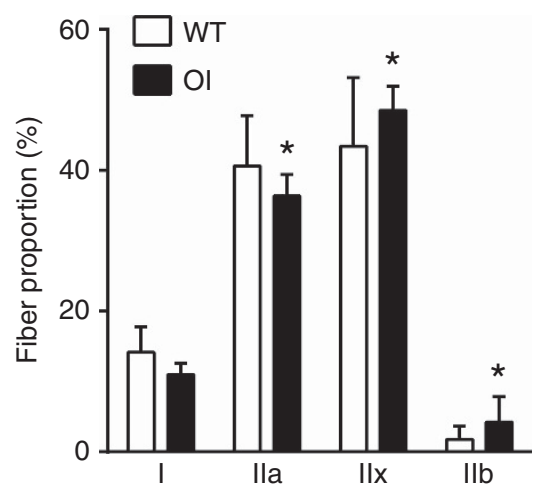

Figure 3. Diaphragm fiber characteristics. Fiber size (cross-sectional area) expressed as the mean value (a), as well as the distribution of fiber sizes in the diaphragm (b), are shown for wild-type (WT) and osteogenesis imperfecta (OI) mice ( $n=6$ per group). The mean fiber size (c) and relative proportions (d), of each of the MyHC-defined fiber types within the diaphragm, are also indicated. Values are mean+SD. ${ }^{*} P<0.05$ for WT vs. OI.
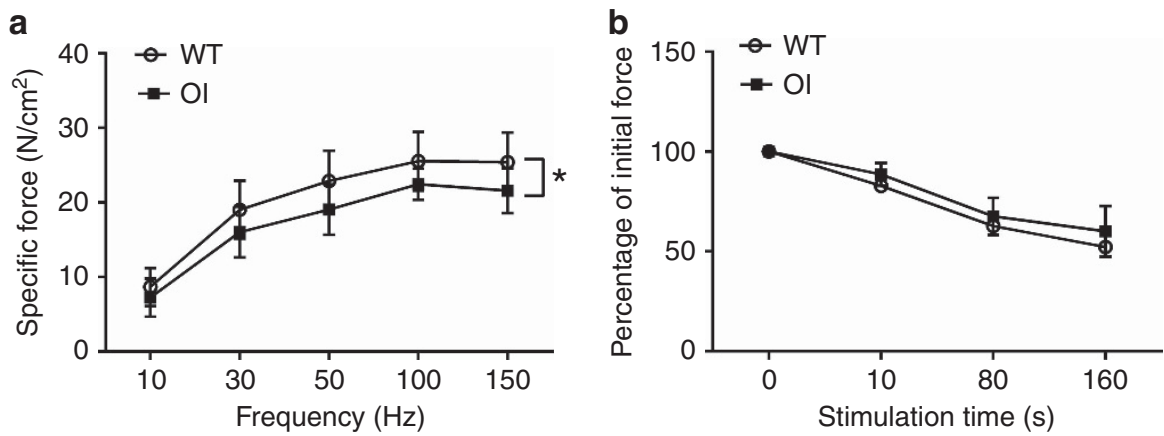

Figure 4. Diaphragm force reduction in osteogenesis imperfecta (OI) mice. (a) Specific force generation (i.e., force normalized to muscle crosssectional area) determined at different stimulation frequencies; and (b) fatigue resistance assessed during repetitive stimulation in vitro. Values are mean+SD ( $n=6$ mice per group). ${ }^{*} P<0.05$ for wild-type (WT) vs. Ol.

\section{Anti-TGF- $\beta$ Treatment does not Prevent Lung Pathology}

The effects of treatment with anti-TGF- $\beta$-neutralizing antibody during the period from 4 to 8 weeks of age are shown in Figure 5. At this earlier age (i.e., 8 weeks old as compared with 14 weeks of age shown in Figure 1), OI mice treated with an isotype control antibody have begun to demonstrate regions with larger distal airspaces compared with WT mice (Figure 5a,b) with a trend toward increased Lm values (Figure 5e). There was no significant prevention of this phenomenon by anti-TGF- $\beta$ treatment (Figures $5 \mathrm{c}, \mathrm{d}$ ), and in fact Lm values were highest in the OI mice that had received anti-TGF- $\beta$ antibody injections (Figure 5e).

\section{DISCUSSION}

Type I collagen is an important component of both pulmonary (16) and skeletal muscle tissue (17). Although respiratory complications of OI are well recognized as being responsible for significant morbidity and mortality in the 


\section{Osteogenesis imperfecta and respiration Articles}
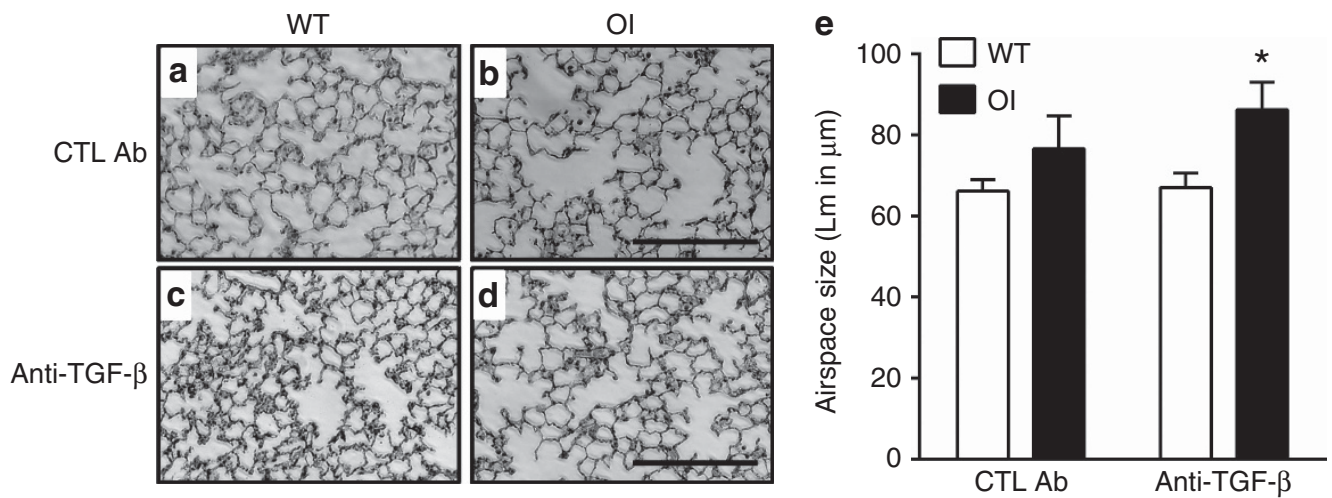

Figure 5. Lack of benefit of anti-transforming growth factor (TGF- $\beta$ ) treatment. Representative lung section images stained with H\&E for mice treated with isotype control (CTL) antibody (a and $\mathbf{b}$ ) or anti-TGF- $\beta$ antibody (c and $\mathbf{d}$ ) for 4 weeks; scale bars $=100 \mu \mathrm{m}$. Airspace size was quantified by Lm (e) in the wild-type (WT) ( $n=9$ of which 4 received anti-TGF- $\beta$ ) and osteogenesis imperfecta (OI) ( $n=7$ of which 3 received anti-TGF- $\beta$ ) groups at 8 weeks of age. Values are mean+SD. ${ }^{*} P<0.05$ for WT vs. Ol.

moderate to severe forms of the disease (3-5), the precise etiology of respiratory impairment in OI is not well defined. The loss of lung function has generally been solely attributed to the effects of spinal and ribcage deformities, resulting from the underlying bone disease. However, our data suggest that other factors may be involved. In this regard, here we report the existence of both abnormal distal airspace enlargement and diaphragm muscle pathology (i.e., reduced mass and specific force) in the Colla1 ${ }^{\text {rtt/ }}$ mouse model of OI.

To date, abnormal pulmonary phenotypes have been described in two other OI mouse models $(6,8)$. In mice with a dominant frameshift mutation in the collagen type I $\alpha 1$ C-propeptide domain (termed Aga2 mice), the lungs exhibited areas of alveolar hemorrhage and inflammation, and this was accompanied by impaired gas exchange (6). However, the Aga2 mice also showed signs of cardiac dysfunction that could have played a role in these changes, and there was no assessment of distal airspace size (6). On the other hand, previous studies in mice that are deficient in CRTAP, a member of the enzymatic complex responsible for 3 -hydroxylation of proline in the $\alpha 1$ chain of type I collagen, showed evidence of distal airspace enlargement similar to our study $(7,8)$.

In patients with Ehlers-Danlos syndrome, which is also linked to mutations in the COL1A1 and COL1A2 genes, the development of bullous emphysema has been well described $(18,19)$. Whereas the distal airspace enlargement associated with emphysema in smokers is largely a consequence of active lung tissue destruction, such abnormalities in Ehlers-Danlos patients or OI mice are presumably a direct consequence of the dysfunctional type I collagen, causing a loss of pulmonary elastic recoil. Interestingly, the Colla1 $1^{\mathrm{Jrt} / \mathrm{T}}$ mouse used in our study, was originally published as a combined model of OI and Ehlers-Danlos syndrome (10). These mice have a typical OI mutation (splice mutation leading to an in-frame deletion in the triple helical domain of the alpha 1 (I) chain) as well as the characteristic OI phenotypic feature of spontaneous fractures. The exaggerated spine curvature and laxity of ligaments as well as skin observed in these mice are also part of the typical OI phenotype. On the other hand, these mice do not demonstrate joint dislocations, ectopia lentis, or a vascular phenotype, which would be the hallmarks of Ehlers-Danlos syndrome. Therefore, we consider the Colla $1^{\mathrm{Jt} / \mathrm{t}}$ mouse to be primarily a model of the more severe forms of OI known to be associated with impaired respiratory function in humans.

A previous study reported that administration of neutralizing antibodies against TGF- $\beta$ prevented the development of pulmonary airspace enlargement in CRTAP-deficient mice (8). However, in contrast to the CRTAP-deficient mouse model (8), we failed to find any mitigation of abnormal airspace enlargement in our Colla $1^{\mathrm{Jtt} / \mathrm{T}}$ mice treated with antiTGF- $\beta$ antibodies. Indeed, there appeared to be greater airspace size in OI mice receiving the anti-TGF- $\beta$ treatment, which is consistent with previous findings of alveolar airspace enlargement in Smad3-null mice that are defective in TGF- $\beta$ signaling (20). The divergent findings in the different OI mouse models most likely reflect the genetic heterogeneity of the disease. In addition, one should note that in the present study, the effects of anti-TGF- $\beta$ antibody treatment were only evaluated in female mice. Although this study was not designed to directly compare males and females at equivalent ages, our findings are consistent with the development of abnormal pulmonary airspace enlargement in OI mice of both sexes.

In skeletal muscle, type I collagen is expressed during development and in adult muscle tissues. It is primarily localized within the epimysial and perimysial extracellular matrix surrounding muscle fibers, as well as within tendinous structures $(17,21)$. In the present study, diaphragmatic thickness was greatly reduced in OI mice. This was primarily the result of a reduction in fiber number rather than atrophy of individual myofibers, although there was a trend toward smaller mean CSA in most fiber types. Given the fact that OI mice were significantly smaller and had reduced body 


\section{Articles | Baglole et al.}

weights, it appears likely that the decreases in the diaphragm muscle thickness and the number were at least partly related to developmental factors, leading to globally stunted body growth. There were also small changes in MyHC phenotype within OI diaphragms, consisting of a slight shift in favor of IIx/IIb fibers. These changes differ from what is observed in human emphysema, where there is a shift toward more fatigue-resistant type I (slow-twitch) fibers in the diaphragm (22). The latter is consistent with a protective adaptation to prevent diaphragmatic fatigue, since patients with emphysema also have increased airways resistance that substantially augments the workload placed on the diaphragm. In contrast, experimental animals with elastase-induced emphysema have an isolated loss of lung elastic recoil with normal pulmonary resistance (23), and under these conditions the changes in the diaphragmatic fiber types are minimally altered (24) as observed in the OI mice of the current study.

From a functional standpoint, the OI diaphragm exhibited a reduction in force generation per unit cross-sectional area of the muscle (referred to as specific force). This decrease in force-generating capacity of the diaphragm was modest but consistent, and implies a reduced ability to compensate for increased respiratory loads that might be encountered during pulmonary infections. The results of our investigation do not allow us to ascertain the precise mechanism underlying the force impairment in the diaphragms of OI mice. However, type I collagen is believed to play an important role in allowing the force generated by contracting sarcomeres to be effectively transmitted to the extracellular matrix and tendon (17). The latter includes the central tendon of the diaphragm, which if excessively compliant could compromise the tension development by the muscle and hence impair negative pressure generation within the thorax. The results of our study are consistent with those of Veilleux et al. (9), who found that children and adolescent patients with OI (type I) had smaller muscle size and lower specific force generation in their lower extremity muscles.

The proposition that respiratory abnormalities in OI are not only due to bone disease is supported by the existing albeit limited human clinical data. For example, pulmonary hypoplasia has been rarely reported in OI patients $(25,26)$, suggesting that defects in lung development could contribute to respiratory impairment. Significant pulmonary impairment has also been reported in OI patients despite little scoliosis being present (27). Similarly, an accelerated decline in pulmonary function over time was noted in OI children before the development of significant spinal deformity (6). Our observations are consistent with the above, as the pulmonary parenchymal changes found in Colla1 ${ }^{\text {Jrt/+ }}$ mice are not restrictive in nature and cannot be accounted for by scoliosis. These findings may have clinical implications to the extent that the current bone-directed therapies for OI would not be expected to address these intrinsic pulmonary and diaphragmatic abnormalities, thus maintaining the OI patients at increased risk for respiratory complications.

\section{STATEMENT OF FINANCIAL SUPPORT}

This study was supported by the Shriners of North America and Deutsche Forschungsgemeinschaft (\#TA1088/1-1), the Canadian Institutes of Health Research, the Fonds de Recherche du Quebec - Santé, the JT Costello Memorial Fund, and the McGill University Health Centre Research Institute.

Disclosure: The authors declare no conflict of interest.

\section{REFERENCES}

1. Trejo P, Rauch F. Osteogenesis imperfecta in children and adolescentsnew developments in diagnosis and treatment. Osteoporos Int 2016;27: 3427-37.

2. Bardai G, Moffatt P, Glorieux FH, et al. DNA sequence analysis in 598 individuals with a clinical diagnosis of osteogenesis imperfecta: diagnostic yield and mutation spectrum. Osteoporos Int 2016;27:3607-13.

3. McAllion SJ, Paterson CR. Causes of death in osteogenesis imperfecta. J Clin Pathol 1996;49:627-30.

4. Wekre LL, Kjensli A, Aasand K, et al. Spinal deformities and lung function in adults with osteogenesis imperfecta. Clin Respir J 2014;8:437-43.

5. Folkestad L, Hald JD, Canudas-Romo V, et al. Mortality and causes of death in patients with osteogenesis imperfecta: a register-based nationwide cohort study. J Bone Miner Res 2016;31:2159-66.

6. Thiele F, Cohrs CM, Flor A, et al. Cardiopulmonary dysfunction in the osteogenesis imperfecta mouse model Aga2 and human patients are caused by bone-independent mechanisms. Hum Mol Genet 2012;21:3535-45.

7. Baldridge $\mathrm{D}$, Lennington $\mathrm{J}$, Weis $\mathrm{M}$, et al. Generalized connective tissue disease in Crtap-/- mouse. PLoS ONE 2010;5:e10560.

8. Grafe I, Yang T, Alexander S, et al. Excessive transforming growth factor $-\beta$ signaling is a common mechanism in osteogenesis imperfecta. Nat Med 2014;20:670-5.

9. Veilleux L-N, Lemay M, Pouliot-Laforte A, Cheung MS, Glorieux FH, Rauch F. Muscle anatomy and dynamic muscle function in osteogenesis imperfecta type I. J Clin Endocrinol Metab 2014;99:E356-62.

10. Chen F, Guo R, Itoh S, et al. First mouse model for combined osteogenesis imperfecta and Ehlers-Danlos syndrome. J Bone Miner Res 2014;29:1412-23.

11. Knudsen L, Weibel ER, Gundersen HJG, Weinstein F V, Ochs M. Assessment of air space size characteristics by intercept (chord) measurement: an accurate and efficient stereological approach. J Appl Physiol 2010;108:412-21.

12. Laucho-Contreras ME, Taylor KL, Mahadeva R, Boukedes SS, Owen CA. Automated measurement of pulmonary emphysema and small airway remodeling in cigarette smoke-exposed mice. J Vis Exp 2015: 52236.

13. Petrof BJ, Stedman HH, Shrager JB, Eby J, Sweeney HL, Kelly AM. Adaptations in myosin heavy chain expression and contractile function in dystrophic mouse diaphragm. Am J Physiol 1993;265:C834-41.

14. Schneider CA, Rasband WS, Eliceiri KW. NIH Image to ImageJ: 25 years of image analysis. Nat Methods 2012;9:671-5.

15. Divangahi M, Matecki S, Dudley RW, et al. Preferential diaphragmatic weakness during sustained Pseudomonas aeruginosa lung infection. Am J Respir Crit Care Med 2004;169:679-86.

16. Burgess JK, Mauad T, Tjin G, Karlsson JC, Westergren-Thorsson G. The extracellular matrix-the under-recognized element in lung disease? J Pathol 2016;240:397-409.

17. Gillies AR, Lieber RL. Structure and function of the skeletal muscle extracellular matrix. Muscle Nerve 2011;44:318-31.

18. Ruggeri P, Calcaterra S, Girbino G. Bullous emphysema as first presentation of Ehlers-Danlos syndrome in monozygotic twins. Respir Med Case Reports 2015;14:40-2.

19. Ayres JG, Pope FM, Reidy JF, Clark TJ. Abnormalities of the lungs and thoracic cage in the Ehlers-Danlos syndrome. Thorax 1985;40:300-5.

20. Bonniaud P, Kolb M, Galt T, et al. Smad3 null mice develop airspace enlargement and are resistant to TGF-beta-mediated pulmonary fibrosis. J Immunol 2004;173:2099-108.

21. Duance VC, Restall DJ, Beard H, Bourne FJ, Bailey AJ. The location of three collagen types in skeletal muscle. FEBS Lett 1977;79:248-52. 
22. Levine S, Kaiser L, Leferovich J, Tikunov B. Cellular adaptations in the diaphragm in chronic obstructive pulmonary disease. N Engl J Med 1997;337:1799-806.

23. Bellofiore S, Eidelman DH, Macklem PT, Martin JG. Effects of elastaseinduced emphysema on airway responsiveness to methacholine in rats. J Appl Physiol 1989;66:606-12.

24. Van Der Heijden HF, Dekhuijzen PN, Folgering H, Ginsel LA, Van Herwaarden CL. Long-term effects of clenbuterol on diaphragm morphology and contractile properties in emphysematous hamsters. J Appl Physiol 1998;85:215-22.
25. Shapiro JR, Burn VE, Chipman SD, et al. Pulmonary hypoplasia and osteogenesis imperfecta type II with defective synthesis of alpha I(1) procollagen. Bone 1989;10:165-71.

26. Thibeault DW, Pettett G, Mabry SM, Rezaiekhaligh MM. Osteogenesis imperfecta Type IIA and pulmonary hypoplasia with normal alveolar development. Pediatr Pulmonol 1995;20:301-6.

27. Widmann RF, Bitan FD, Laplaza FJ, Burke SW, DiMaio MF, Schneider R. Spinal deformity, pulmonary compromise, and quality of life in osteogenesis imperfecta. Spine (Phila PA 1976) 1999;24: $1673-8$. 